\title{
Data-driven output synchronization of heterogeneous leader-follower multi-agent systems*
}

\author{
Junjie Jiao $^{1}$, Henk J. van Waarde ${ }^{2}$, Harry L. Trentelman ${ }^{3}$, M. Kanat Camlibel ${ }^{3}$, and Sandra Hirche ${ }^{1}$
}

\begin{abstract}
This paper deals with data-driven output synchronization for heterogeneous leader-follower linear multiagent systems. Given a multi-agent system that consists of one autonomous leader and a number of heterogeneous followers with external disturbances, we provide necessary and sufficient data-based conditions for output synchronization. We also provide a design method for obtaining such output synchronizing protocols directly from data. The results are then extended to the special case that the followers are disturbance-free. Finally, a simulation example is provided to illustrate our results.
\end{abstract}

\section{INTRODUCTION}

Over the last two decades, the design of distributed protocols for multi-agent systems that achieve consensus or synchronization has been an active research topic in the field of systems and control, see e.g., [1]-[7]. Most of the existing work is concerned with model-based approaches, i.e. they assume that agent models are known. In particular, it is shown in [2] that solvability of certain regulator equations is a necessary condition for output synchronization, and suitable protocols are proposed.

To remove the dependency on agent models in consensus or synchronization problems, some existing papers propose data-driven approaches based on reinforcement learning. In [8], a data-based adaptive dynamic programming method is proposed for computing optimal distributed control algorithms for leader-follower multi-agent systems. In [9], a synchronization problem is first interpreted as a multi-agent discrete-time dynamic game, and then distributed protocols are proposed based on reinforcement learning value iteration algorithms. However, these data-driven methods require large amounts of data and are computationally expensive.

Very recently, based on Willems' fundamental lemma [10], the problem of system analysis and control directly from data has attracted much attention, see e.g. [11]-[14]. In [11], several control problems are solved directly from data. Later

*The work of J. Jiao and S. Hirche has received funding from the German Research Foundation (DFG) within the Joint Sino-German research project Control and Optimization for Event-triggered Networked Autonomous Multi-agent Systems (COVEMAS). The work of H. J. van Waarde has received funding from the European Research Council under the Advanced ERC Grant Agreement Switchlet n. 670645.

${ }^{1}$ J. Jiao and S. Hirche are with the Chair of Information-oriented Control, Department of Electrical and Computer Engineering, Technical University of Munich, 80333, Munich, Germany. Email: junjie.jiao@tum.de; hircheetum. de

${ }^{2} \mathrm{H}$. J. van Waarde is with the Control Group, Department of Engineering, University of Cambridge, Cambridge, UK. Email: hv280@ cam.ac.uk

${ }^{3} \mathrm{H}$. L. Trentelman and M. K. Camlibel are with the Bernoulli Institute for Mathematics, Computer Science, and Artificial Intelligence, University of Groningen, Nijenborgh 9, 9747 AG, Groningen, The Netherlands. Email: h.l.trentelmanerug.nl; m.k.camlibelerug.nl on, several fundamental analysis and control problem are addressed in [13], without the requirement of persistency of excitation. A robust data-driven design is proposed in [15] for computing feedback controllers directly using (noisy) data. In [14], several versions of the S-Lemma are generalized to matrix versions, which are then used to design feedback controllers from noisy data. For more work on this topic, we refer to [12], [16], [17] and the references therein.

While the majority of research on data-driven control has focused on centralized settings for single systems, the distributed setting for networked systems is relatively unexplored. In [18], a distributed data-based predictive control method is proposed to stabilize networked systems. In [19], the problem of synthesizing distributed data-based controllers from noiseless data is considered. Using noisy input-state data, guaranteed $H_{\infty}$ performance analysis and controller synthesis are provided in [20] for interconnected systems. For further related work, see also [21], [22].

Different from the above work [18]-[22], in the present paper, we will deal with the data-driven output synchronization problem for heterogeneous leader-follower multi-agent systems. In particular, we will provide data-based conditions under which the proposed distributed protocols achieve output synchronization for multi-agent systems. We will also provide a method for computing such output synchronizing protocols directly from data.

This paper is organized as follows. In Section [II we introduce some notation and graph theory. In Section [II] we formulate the data-driven output synchronization problem. In order to solve the formulated problem, in Section IV we review some relevant results on model-based output synchronization and data informativity for stabilization by state feedback. in Section $\nabla$ we solve the problems formulated in Section III To illustrate our proposed method, a simulation example is given in Section VI] Finally, Section VII concludes this paper.

\section{NOTATION AND GRAPH THEORY}

\section{A. Notation}

We denote by $\mathbb{R}$ the field of real numbers, and by $\mathbb{R}^{n}$ the $n$-dimensional real Euclidean space. We denote by $\mathbb{R}^{n \times m}$ the space of real $n \times m$ matrices. For a given matrix $A$, its transpose is denoted by $A^{\top}$. By $\operatorname{diag}\left(a_{1}, a_{2}, \ldots, a_{n}\right)$, we denote the $n \times n$ diagonal matrix with $a_{1}, a_{2}, \ldots, a_{n}$ on the diagonal. For a linear map $A: \mathscr{X} \rightarrow \mathscr{Y}$, the image and kernel of $A$ are denoted by $\operatorname{im}(A):=\{A x \mid x \in \mathscr{X}\}$ and $\operatorname{ker}(A):=\{x \in \mathscr{X} \mid A x=0\}$. 


\section{B. Graph theory}

A weighted directed graph is denoted by $\mathscr{G}=(\mathscr{V}, \mathscr{E}, \mathscr{A})$, where $\mathscr{V}=\{1,2, \ldots, N\}$ is the finite nonempty node set, $\mathscr{E} \subset$ $\mathscr{V} \times \mathscr{V}$ is the edge set of ordered pairs $(i, j)$ and $\mathscr{A}=\left[a_{i j}\right]$ is the associated adjacency matrix with nonnegative entries. The entry $a_{j i}$ of the adjacency matrix $\mathscr{A}$ is the weight associated with the edge $(i, j)$ and $a_{j i}$ is nonzero if and only if $(i, j) \in \mathscr{E}$. A graph is called simple if $a_{i i}=0$, i.e. the graph does not contain self-loops. A directed tree is a directed graph in which one node (called the root node) has its in-degree equal to zero and all other nodes have their indegree equal to one. A spanning tree of a directed graph $\mathscr{G}$ is a directed tree that connects all nodes of the graph $\mathscr{G}$.

Given a graph $\mathscr{G}$, the degree matrix of $\mathscr{G}$ is denoted by $\mathscr{D}=\operatorname{diag}\left(d_{1}, d_{2}, \ldots, d_{N}\right)$ with $d_{i}=\sum_{j=1}^{N} a_{i j}$. The Laplacian matrix of $\mathscr{G}$ is defined as $L:=\mathscr{D}-\mathscr{A}$. If $\mathscr{G}$ is a weighted directed graph, its Laplacian matrix $L$ has at least one zero eigenvalue associated with the eigenvector $\mathbf{1}_{N}$ and all nonzero eigenvalues have positive real parts. Furthermore, zero is a simple eigenvalue of $L$ if and only if $\mathscr{G}$ contains a spanning tree.

\section{PROBLEM FORMULATION}

We consider a leader-follower multi-agent system that consists of one leader and $N$ heterogeneous followers. The dynamics of the leader is represented by

$$
x_{r}(k+1)=S x_{r}(k),
$$

and is assumed to be known, where $x_{r} \in \mathbb{R}^{r}$ and $S \in \mathbb{R}^{r \times r}$. It is reasonable to assume that the leader dynamics is known, since the followers need to know the dynamics they will synchronize on. The dynamics of the $i$ th follower $(i=$ $1,2, \ldots, N)$ is described by

$$
x_{i}(k+1)=\bar{A}_{i} x_{i}(k)+\bar{B}_{i} u_{i}(k)+\bar{E}_{i} w_{i}(k),
$$

where $x_{i} \in \mathbb{R}^{n_{i}}$ is the state of the $i$ th follower, $u_{i} \in \mathbb{R}^{m_{i}}$ is the associated control input, and $w_{i} \in \mathbb{R}^{q_{i}}$ the external disturbance. The matrices $\bar{A}_{i}, \bar{B}_{i}$ and $\bar{E}_{i}$ are of suitable dimensions. We refer to (2) as the 'true' system of the $i$ th follower, denoted by $\left(\bar{A}_{i}, \bar{B}_{i}, \bar{E}_{i}\right)$. We consider the situation that the matrices $\bar{A}_{i}, \bar{B}_{i}$ and $\bar{E}_{i}$ of the true system are unknown and we only have access to a finite set of data on the finite time interval $\{0,1, \ldots, \tau\}$, generated by the followers, namely,

$$
\begin{aligned}
& U_{i-}:=\left[\begin{array}{llll}
u_{i}(0) & u_{i}(1) & \cdots & u_{i}(\tau-1)
\end{array}\right], \\
& X_{i}:=\left[\begin{array}{llll}
x_{i}(0) & x_{i}(1) & \cdots & x_{i}(\tau)
\end{array}\right]
\end{aligned}
$$

as well as measurements of the disturbances

$$
W_{i-}=\left[\begin{array}{llll}
w_{i}(0) & w_{i}(1) & \cdots & w_{i}(\tau-1)
\end{array}\right] .
$$

By partitioning the state data as

$$
\begin{aligned}
& X_{i-}=\left[\begin{array}{llll}
x_{i}(0) & x_{i}(1) & \cdots & x_{i}(\tau-1)
\end{array}\right], \\
& X_{i+}=\left[\begin{array}{llll}
x_{i}(1) & x_{i}(2) & \cdots & x_{i}(\tau)
\end{array}\right]
\end{aligned}
$$

we can relate the data and the true system $\left(\bar{A}_{i}, \bar{B}_{i}, \bar{E}_{i}\right)$ of the $i$ th follower through

$$
X_{i+}=\left[\begin{array}{lll}
\bar{A}_{i} & \bar{B}_{i} & \bar{E}_{i}
\end{array}\right]\left[\begin{array}{l}
X_{i-} \\
U_{i-} \\
W_{i-}
\end{array}\right] .
$$

Note that the true system $\left(\bar{A}_{i}, \bar{B}_{i}, \bar{E}_{i}\right)$ may not be the only system that explains the data $\left(U_{i-}, W_{i-}, X_{i}\right)$ of the $i$ th follower, see e.g., [13]. Therefore, we define the set of all systems $\left(A_{i}, B_{i}, E_{i}\right)$ that explain the data $\left(U_{i-}, W_{i-}, X_{i}\right)$ of the $i$ th follower by

$$
\Sigma_{w, i}:=\left\{\left(A_{i}, B_{i}, E_{i}\right) \mid X_{i+}=\left[\begin{array}{lll}
A_{i} & B_{i} & E_{i}
\end{array}\right]\left[\begin{array}{l}
X_{i-} \\
U_{i-} \\
W_{i-}
\end{array}\right]\right\} .
$$

Obviously, $\left(\bar{A}_{i}, \bar{B}_{i}, \bar{E}_{i}\right) \in \Sigma_{w, i}$.

In this paper, we consider the output synchronization problem [2], [23], [24]. To this end, we assign to the leader (1) an output

$$
y_{r}(k)=R x_{r}(k),
$$

where $y_{r} \in \mathbb{R}^{p}$, and $R \in \mathbb{R}^{p \times r}$ is a known matrix. We assume that the pair $(R, S)$ is observable. We also assign to each follower (2) an output

$$
y_{i}(k)=C_{i} x_{i}(k)+D_{i} u_{i}(k),
$$

where $y_{i} \in \mathbb{R}^{p}$, and the matrices $C_{i}$ and $D_{i}$ are known matrices that specify the outputs to be synchronized.

We also make the following standard assumption for output synchronization [23], [25], [26]:

Assumption 1: We assume that all eigenvalues of $S$ are simple and lie on the unit circle.

Following [23], we consider the case that the followers (2) will be interconnected by a distributed protocol of the form

$$
\begin{aligned}
v_{i}(k+1) & =S v_{i}(k)+\left(1+d_{i}+g_{i}\right)^{-1} F \\
& \times\left(\sum_{j=1}^{N} a_{i j}\left(v_{j}(k)-v_{i}(k)\right)+g_{i}\left(x_{r}(k)-v_{i}(k)\right)\right), \\
u_{i}(k) & =K_{i}\left(x_{i}(k)-\Pi_{i} v_{i}(k)\right)+\Gamma_{i} v_{i}(k), \quad i=1,2, \ldots, N
\end{aligned}
$$

where $v_{i} \in \mathbb{R}^{r}$ is the state of the $i$ th local controller, $F$ and $K_{i}$ are gain matrices to be designed, $\Pi_{i}$ and $\Gamma_{i}$ are matrices to be determined later. The coefficient $a_{i j}$ is the $i j$ th entry of the adjacency matrix $\mathscr{A}$ of the communication graph between the followers, $d_{i}$ is the node degree of the $i$ th follower, and $g_{i}$ represents the communication between the leader and the followers. Strict inequality $g_{i}>0$ means that the leader shares its state information with the $i$ th follower, otherwise $g_{i}=0$.

Definition 1: The protocol (8) is said to achieve output synchronization for the multi-agent system (1) and (2) if $y_{i}(k)-y_{r}(k) \rightarrow 0, v_{i}(k)-x_{r}(k) \rightarrow 0$ and $x_{i}(k)-\Pi_{i} v_{i}(k) \rightarrow 0$ as $k \rightarrow \infty$.

We make the following standing assumption regarding the communication between the leader and followers [23], [27].

Assumption 2: We assume that the communication graph between the followers is a simple directed graph that contains 
a spanning tree, and the leader shares its information with at least one of the root nodes.

Recall that we only have access to a finite set of data $\left(U_{i-}, W_{i-}, X_{i}\right)$ of the followers. Since the data of each follower can be explained by a set of systems as defined in (5), and it is not possible to distinguish the true system (2) from other systems in this set. We then introduce the following definition:

Definition 2: The data $\left(U_{i-}, W_{i-}, X_{i}\right)$ are informative for output synchronization if there exists a protocol (8) that achieves output synchronization for the leader (1) and all systems $\left(A_{i}, B_{i}, E_{i}\right) \in \Sigma_{w, i}$, for $i=1,2, \ldots, N$.

The problem that we want to address is the following.

Problem 1: Find conditions under which the data $\left(U_{i-}, W_{i-}, X_{i}\right)$ are informative for output synchronization. Also, provide a design method for computing a protocol (8) that achieves output synchronization.

Before solving Problem 11 we will first review some relevant results on model-based output synchronization and data informativity for stabilization. These preliminary results are necessary ingredients for addressing Problem 1

\section{PReliminary Results}

\section{A. Model-based output synchronization of heterogeneous leader-follower multi-agent systems}

In this subsection, we will review some relevant results on model-based output synchronization of heterogeneous leaderfollower linear multi-agent systems, see also [23].

Consider a multi-agent system that consists of one leader and $N$ heterogeneous followers. The dynamics of the leader is represented by (1) with associated output (6). We assume that Assumption 1 holds. The dynamics of the $i$ th follower is represented by

$$
x_{i}(k+1)=A_{i} x_{i}(k)+B_{i} u_{i}(k), \quad i=1,2, \ldots, N
$$

with associated output (7). All system matrices are assumed to be known. We also assume that the pairs $\left(A_{i}, B_{i}\right)$ are stabilizable and the pairs $\left(C_{i}, A_{i}\right)$ are detectable.

Following [23], we consider the case that the followers (9]) are to be interconnected by a distributed protocol of the form (8). We also assume that Assumption 2 holds. The aim is then to design a protocol (8) such that the controlled multi-agent system achieves output synchronization.

The following proposition provides necessary and sufficient conditions under which protocols (8) achieve output synchronization, see also [23].

Proposition 1: Let Assumptions 1 and 2 hold. Let $F$ and $K_{i}$ be matrices such that $S-\lambda_{i} F$ and $A_{i}+B_{i} K_{i}, i=1,2, \ldots, N$ are stable, where $\lambda_{i}$ are the eigenvalues of the matrix $\left(I_{N}+\right.$ $\mathscr{D}+G)^{-1}(L+G)$ with $G=\operatorname{diag}\left(g_{1}, g_{2}, \ldots, g_{N}\right)$. Then there exists a protocol (8) that achieves output synchronization for the multi-agent system (1) and (9) if and only if there exist matrices $\Pi_{i} \in \mathbb{R}^{n_{i} \times r}$ and $\Gamma_{i} \in \mathbb{R}^{m_{i} \times r}$ satisfying the regulator equations

$$
\begin{aligned}
& A_{i} \Pi_{i}+B_{i} \Gamma_{i}=\Pi_{i} S, \\
& C_{i} \Pi_{i}+D_{i} \Gamma_{i}=R, \quad i=1,2, \ldots, N .
\end{aligned}
$$

The proof of Proposition 1 is similar to the results in [23] and is omitted here. Note that the system equation (7) representing the output of the $i$ th follower is slightly more general than that in [23]. Indeed, the output equations in our followers contain a direct feed-through term.

Remark 1: We note that there exist methods to compute a simultaneously stabilizing gain matrix $F$ in the sense that $S-\lambda_{i} F$ is stable for $i=1,2, \ldots, N$. For instance, in [27], such a gain matrix $F$ is computed by solving discrete-time Riccati inequalities.

\section{B. Data-driven stabilization by state feedback for linear systems}

In this subsection, we will review some results from [28] and [13] on data-driven stabilization by state feedback for linear systems.

Consider the linear system

$$
x(k+1)=\bar{A} x(k)+\bar{B} u(k)+\bar{E} w(k),
$$

where $x \in \mathbb{R}^{n}$ is the state, $u \in \mathbb{R}^{m}$ the input and $w \in \mathbb{R}^{q}$ the external disturbance. The matrices $\bar{A}, \bar{B}$ and $\bar{E}$ are of suitable dimensions. We consider the case that the dynamics of the system (12) is unknown, i.e., the matrices $\bar{A}, \bar{B}$ and $\bar{E}$ are unknown. However, similar to (3) and (4), we assume that we have access to a finite set of data of system (12), namely, $\left(U_{-}, W_{-}, X\right)$.

We refer to (12) as the 'true' system, denoted by $(\bar{A}, \bar{B}, \bar{E})$. Note that the true system $(\bar{A}, \bar{B}, \bar{E})$ may not be the only system that explains the data $\left(U_{-}, W_{-}, X\right)$, see e.g. [13], [28]. To this end, we define the set of all systems $(A, B, E)$ that explain the data $\left(U_{-}, W_{-}, X\right)$ by

$$
\Sigma_{w}:=\left\{(A, B, E) \mid X_{+}=\left[\begin{array}{lll}
A & B & E
\end{array}\right]\left[\begin{array}{l}
X_{-} \\
U_{-} \\
W_{-}
\end{array}\right]\right\} .
$$

Clearly, $(\bar{A}, \bar{B}, \bar{E}) \in \Sigma_{w}$.

In what follows, we will consider the problem of finding a stabilizing controller for the system (12), using only and directly the data $\left(U_{-}, W_{-}, X\right)$. For this, we introduce the following notion of informativity for stabilization by state feedback.

Definition 3: We say that the data $\left(U_{-}, W_{-}, X\right)$ are informative for stabilization by state feedback if there exists a gain $K$ such that $A+B K$ is stable, for all $(A, B, E) \in \Sigma_{w}$.

The follow proposition provides necessary and sufficient conditions for informativity for stabilization by state feedback, see also [28, Lemma 12].

Proposition 2: The data $\left(U_{-}, W_{-}, X\right)$ are informative for stabilization by state feedback if and only if the matrix $X_{-}$ has full row rank and there exists a right inverse $X_{-}^{\dagger}$ of $X_{-}$ such that $X_{+} X_{-}^{\dagger}$ is stable and $W_{-} X_{-}^{\dagger}=0$. Moreover, $K$ is such that $A+B K$ is stable for all $(A, B, E) \in \Sigma_{w}$ if and only if $K=U_{-} X_{-}^{\dagger}$, where $X_{-}^{\dagger}$ satisfies the above properties.

Similar results can also be obtained for the special case that in (12) the external disturbance $w=0$, i.e. the system (12) is disturbance-free [13, Theorem 16]. 


\section{DATA-DRIVEN OUTPUT SYNCHRONIZATION FOR MULTI-AGENT SYSTEMS}

In this section, we will address Problem 1 More specifically, we will provide necessary and sufficient conditions under which the data $\left(U_{i-}, W_{i-}, X_{i}\right)$ of the followers (2) are informative for output synchronization, and we will also provide a design method for computing protocols (8) directly from data that achieve output synchronization.

Before proceeding, we first introduce the following notion of data informativity.

Definition 4: The data $\left(U_{i-}, W_{i-}, X_{i}\right)$ are informative for output regulation if there exist common solutions $\Pi_{i}$ and $\Gamma_{i}$ to (10) and (11) for all $\left(A_{i}, B_{i}, E_{i}\right) \in \Sigma_{w, i}, i=1,2, \ldots, N$.

In the following lemma, we provide necessary and sufficient conditions under which the data $\left(U_{i-}, W_{i-}, X_{i}\right)$ are informative for output regulation.

Lemma 3: Suppose that the data $\left(U_{i-}, W_{i-}, X_{i}\right)$ of the followers (2) are informative for stabilization by state feedback, respectively. Then the data $\left(U_{i-}, W_{i-}, X_{i}\right)$ are informative for output regulation if and only if there exist matrices $M_{i}$ satisfying the linear equations

$$
\begin{aligned}
X_{i+} M_{i}-X_{i-} M_{i} S & =0, \\
W_{i-} M_{i} & =0, \\
C_{i} X_{i-} M_{i}+D_{i} U_{i-} M_{i} & =R, \quad i=1,2, \ldots, N .
\end{aligned}
$$

Proof: Suppose that the data $\left(U_{i-}, W_{i-}, X_{i}\right)$ are informative for stabilization by state feedback, and let $K_{i}$ be a feedback gain such that $A_{i}+B_{i} K_{i}$ is stable for all $\left(A_{i}, B_{i}, E_{i}\right) \in$ $\Sigma_{w, i}$. Then it follows from Proposition 2 that the feedback gain $K_{i}$ can be taken as $K_{i}=U_{i-} X_{i-}^{\dagger}$, where $X_{i-}^{\dagger}$ is a right inverse of $X_{i-}$ such that $X_{i+} X_{i-}^{\dagger}$ is stable and $W_{i-} X_{i-}^{\dagger}=0$. Note that

$$
A_{i}+B_{i} K_{i}=X_{i+} X_{i-}^{\dagger}
$$

$(\Leftarrow)$ Suppose that there exist solutions $M_{i}$ to the equations (14), (15) and (16). Define $\Pi_{i}=X_{i-} M_{i}$ and $\Gamma_{i}=U_{i-} M_{i}$, and take $K_{i}=U_{i-} X_{i-}^{\dagger}$. Recall that the matrices $A_{i}, B_{i}$ and $E_{i}$ satisfy the condition (5) for all $\left(A_{i}, B_{i}, E_{i}\right) \in \Sigma_{w, i}$, the equations (14) can then be written as

$$
\begin{aligned}
\left(A_{i} X_{i-}+B_{i} U_{i-}+E_{i} W_{i-}\right) M_{i}-X_{i-} M_{i} S & =0 \\
\Leftrightarrow \quad A_{i} X_{i-} M_{i}+B_{i} U_{i-} M_{i}-X_{i-} M_{i} S & =0 \\
\Rightarrow \quad A_{i} \Pi_{i}+B_{i} \Gamma_{i}-\Pi_{i} S & =0
\end{aligned}
$$

where we have used (15). It follows that 10 has common solutions $\Pi_{i}$ and $\Gamma_{i}$ for all $\left(A_{i}, B_{i}, E_{i}\right) \in \Sigma_{w, i}$.

For equations (16), use again $\Pi_{i}=X_{i-} M_{i}, \Gamma_{i}=U_{i-} M_{i}$ and $K_{i}=U_{i-} X_{i-}^{\dagger}$, it follows that $\Pi_{i}$ and $\Gamma_{i}$ are also solutions of (11) for all $\left(A_{i}, B_{i}, E_{i}\right) \in \Sigma_{w, i}$.

$(\Rightarrow)$ Suppose that the data $\left(U_{i-}, W_{i-}, X_{i}\right)$ are informative for output regulation, then according to Definition 4 there exist common solutions $\Pi_{i}$ and $\Gamma_{i}$ to (10) and (11) for all $\left(A_{i}, B_{i}, E_{i}\right) \in \Sigma_{w, i}, i=1,2, \ldots, N$.

Define

$$
\Sigma_{w, i}^{0}=\left\{\left(A_{i 0}, B_{i 0}, E_{i 0}\right) \mid 0=\left[\begin{array}{lll}
A_{i 0} & B_{i 0} & E_{i 0}
\end{array}\right]\left[\begin{array}{l}
X_{i-} \\
U_{i-} \\
W_{i-}
\end{array}\right]\right\}
$$

It has been shown in the proof of [28, Lemma 12] that $A_{i 0}+B_{i 0} K_{i}=0$ for all $\left(A_{i 0}, B_{i 0}, E_{i 0}\right) \in \Sigma_{w, i}^{0}$. Subsequently, according to the definitions of $\Sigma_{w, i}$ and $\Sigma_{w, i}^{0}$, we have $\left(A_{i}+\right.$ $\left.A_{i 0}, B_{i}+B_{i 0}, E_{i}\right) \in \Sigma_{w, i}$. Since (10) and (11) have common solutions for all $\left(A_{i}, B_{i}, E_{i}\right) \in \Sigma_{w, i}$, then $\left(A_{i}+A_{i 0}, B_{i}+B_{i 0}, E_{i}\right)$ also satisfies (10) and (11). This implies that

$$
\left(A_{i}+A_{i 0}\right) \Pi_{i}+\left(B_{i}+B_{i 0}\right) \Gamma_{i}=\Pi_{i} S .
$$

Since also (10) holds, it follows that

$$
\left[\begin{array}{lll}
A_{i 0} & B_{i 0} & E_{i 0}
\end{array}\right]\left[\begin{array}{c}
\Pi_{i} \\
\Gamma_{i} \\
0
\end{array}\right]=0
$$

for all $\left(A_{i 0}, B_{i 0}, E_{i 0}\right) \in \Sigma_{w, i}^{0}$. This implies that

$$
\operatorname{ker}\left[\begin{array}{lll}
X_{i-}^{\top} & U_{i-}^{\top} & W_{i-}^{\top}
\end{array}\right] \subseteq \operatorname{ker}\left[\begin{array}{lll}
\Pi_{i}^{\top} & \Gamma_{i}^{\top} & 0
\end{array}\right],
$$

which is equivalent to

$$
\operatorname{im}\left[\begin{array}{c}
\Pi_{i} \\
\Gamma_{i} \\
0
\end{array}\right] \subseteq \operatorname{im}\left[\begin{array}{l}
X_{i-} \\
U_{i-} \\
W_{i-}
\end{array}\right]
$$

As a consequence, there exists matrices $M_{i}$ such that

$$
\left[\begin{array}{c}
\Pi_{i} \\
\Gamma_{i} \\
0
\end{array}\right]=\left[\begin{array}{l}
X_{i-} \\
U_{i-} \\
W_{i-}
\end{array}\right] M_{i}, \quad i=1,2, \ldots, N
$$

By substituting (18), (17) and (5) into (10) and (11), we obtain (14), (15) and (16). This completes the proof.

Remark 2: Note that Lemma 3 considers a version of the output regulation problem with known disturbances, which is slightly different from the results in [29, Theorem 8]. Although the proof of Lemma 3 is similar to that of [29, Theorem 8], we include a proof to make this paper selfcontained.

Based on Lemma 3, we obtain the following main result.

Theorem 4: Let Assumptions 1 and 2 hold. Then the data $\left(U_{i-}, W_{i-}, X_{i}\right)$ are informative for output synchronization if and only if, for $i=1,2, \ldots, N$, the following two statements hold:

1) There exists a right-inverse $X_{i-}^{\dagger}$ of $X_{i-}$ such that $X_{i+} X_{i-}^{\dagger}$ is stable and $W_{i-} X_{i-}^{\dagger}=0$;

2) there exist matrices $M_{i}$ satisfying the linear equations (14), (15) and (16).

In this case, a protocol (8) can be found as follows: take $F$ such that $S-\lambda_{i} F$ are stable, where $\lambda_{i}$ are the eigenvalues of $\left(I_{N}+\mathscr{D}+G\right)^{-1}(L+G)$, and define $K_{i}=U_{i-} X_{i-}^{\dagger}, \Pi_{i}=X_{i-} M_{i}$ and $\Gamma_{i}=U_{i-} M_{i}$.

Proof: Suppose Assumption 2 hold. It then follows directly from Lemma 3, Proposition 2 and Definition 2 that the data $\left(U_{i-}, W_{i-}, X_{i}\right)$ are informative for output synchronization if and only if the data $\left(U_{i-}, W_{i-}, X_{i}\right)$ are informative for stabilization by state feedback and informative for output regulation. Recalling Definitions 3 and 4 the first part of this theorem is then proven.

Next, it follows from Lemma 3, Proposition 2 and Proposition 1 that an output synchronizing protocol 8 can be found 
by taking $K_{i}=U_{i-} X_{i-}^{\dagger}, \Pi_{i}=X_{i-} M_{i}, \Gamma_{i}=U_{i-} M_{i}$, and taking $F$ such that $S-\lambda_{i} F$ are stable, where $\lambda_{i}$ are the eigenvalues of $\left(I_{N}+\mathscr{D}+G\right)^{-1}(L+G)$.

Again, we note that there exist methods to compute $F$ such that $S-\lambda_{i} F$ is stable for $i=1,2, \ldots, N$. For instance, in [27] such a simultaneous stabilizing gain $F$ is obtained by solving discrete-time Riccati inequalities.

In the sequel, we turn our attention to the special case that in (2) the external disturbance $w_{i}=0$, i.e., the multiagent system is disturbance-free. We say that data $\left(U_{i-}, X_{i}\right)$ are informative for output regulation if there exist common solutions $\Pi_{i}$ and $\Gamma_{i}$ to (10) and (11) for all $\left(A_{i}, B_{i}\right) \in \Sigma_{i}$, $i=1,2, \ldots, N$. We will again provide necessary and sufficient conditions under which the data $\left(U_{i-}, X_{i}\right)$ are informative for output synchronization, and we will also provide a design method for computing protocols (8) directly from data that achieve output synchronization.

The following lemma states under what conditions the data $\left(U_{i-}, X_{i}\right)$ are informative for output regulation.

Lemma 5: Suppose that the data $\left(U_{i-}, X_{i}\right)$ are informative for stabilization by state feedback. Then the data are informative for output regulation if and only if there exist matrices $M_{i}$ satisfying the linear equations

$$
\begin{aligned}
X_{i+} M_{i}-X_{i-} M_{i} S & =0, \\
C_{i} X_{i-} M_{i}+D_{i} U_{i-} M_{i} & =R, \quad i=1,2, \ldots, N .
\end{aligned}
$$

Lemma 5 is a direct consequence of Lemma 3 by letting $W_{i-}=0$. Based on Lemma 5] we have the following result.

Proposition 6: Let Assumptions 1 and 2 hold. The data $\left(U_{i-}, X_{i}\right)$ are informative for output synchronization if and only if, for $i=1,2, \ldots, N$, there exists a right-inverse $X_{i-}^{\dagger}$ of $X_{i-}$ such that $X_{i+} X_{i-}^{\dagger}$ is stable, and, in addition, there exist matrices $M_{i}$ satisfying the linear equations (19) and 20).

In this case, a protocol (8) can be found as follows: take $F$ such that $S-\lambda_{i} F$ are stable, where $\lambda_{i}$ are the eigenvalues of $\left(I_{N}+\mathscr{D}+G\right)^{-1}(L+G)$, and define $K_{i}=U_{i-} X_{i-}^{\dagger}, \Pi_{i}=X_{i-} M_{i}$ and $\Gamma_{i}=U_{i-} M_{i}$.

The proof of Proposition 6 follows directly from Theorem 4 and is omitted here.

\section{ILLUSTRATIVE EXAMPLE}

In this section, we will use a simulation example to illustrate our protocols proposed in Proposition 6. Consider a disturbance-free leader-follower multi-agent system, consisting of one leader and nine followers. The dynamic of the leader is given by (1) and (6), where

$$
S=\left[\begin{array}{ll}
0 & 1 \\
1 & 0
\end{array}\right], \quad R=\left[\begin{array}{ll}
1 & 0
\end{array}\right]
$$

The pair $(R, S)$ is observable. By letting the initial state $x_{r 0}=$ $\left[\begin{array}{ll}1 & 1\end{array}\right]^{\top}$, the output of the leader is a constant $y_{r}=1$. The 'true' dynamics of the nine followers are unknown but are

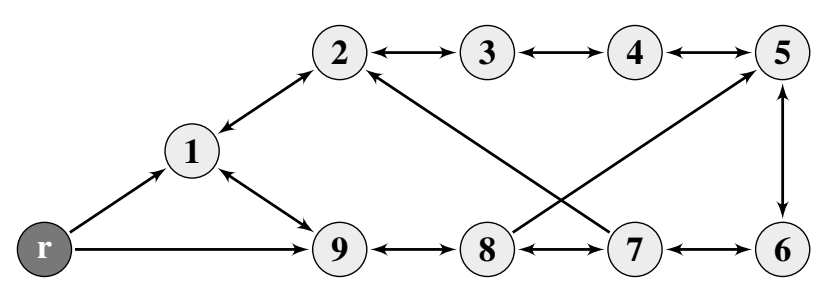

Fig. 1. The underlying graph of the communication between the leader and the followers.

represented by (2) with $\bar{E}_{i}=0$ and (7) with

$$
\begin{aligned}
& \bar{A}_{1}=\bar{A}_{4}=\bar{A}_{7}=\left[\begin{array}{ll}
0 & 1 \\
1 & 1
\end{array}\right], \quad \bar{B}_{1}=\bar{B}_{4}=\bar{B}_{7}=\left[\begin{array}{l}
1 \\
0
\end{array}\right], \\
& C_{1}=C_{4}=C_{7}=\left[\begin{array}{cc}
1 & 1
\end{array}\right], \quad D_{1}=D_{4}=D_{7}=2, \\
& \bar{A}_{2}=\bar{A}_{5}=\bar{A}_{8}=\left[\begin{array}{cc}
0 & 1 \\
1 & -1
\end{array}\right], \quad \bar{B}_{2}=\bar{B}_{5}=\bar{B}_{8}=\left[\begin{array}{l}
1 \\
0
\end{array}\right], \\
& C_{2}=C_{5}=C_{8}=\left[\begin{array}{cc}
-1 & 1
\end{array}\right], \quad D_{2}=D_{5}=D_{8}=2, \\
& \bar{A}_{3}=\bar{A}_{6}=\bar{A}_{9}=\left[\begin{array}{cc}
0 & -1 \\
1 & 0
\end{array}\right], \quad \bar{B}_{3}=\bar{B}_{6}=\bar{B}_{9}=\left[\begin{array}{l}
1 \\
0
\end{array}\right], \\
& C_{3}=C_{6}=C_{9}=\left[\begin{array}{ll}
0 & 1
\end{array}\right], \quad D_{3}=D_{6}=D_{9}=0.5 .
\end{aligned}
$$

It is easy to check that the regulator equations (10) and (11) have solutions for the matrices $\bar{A}_{i}, \bar{B}_{i}, C_{i}$ and $D_{i}$ for $i=1,2, \ldots, 9$. The multi-agent system will be interconnected by a protocol of the form (8). We assume that the communication graph between the agents is given as in Figure 1. The underlying graph between the leader and the followers satisfies Assumption 2.

For each follower, we collect four sets of state data and three sets of input data as follows

$$
\begin{aligned}
& X_{1}=X_{4}=X_{7}=\left[\begin{array}{cccc}
1 & 0 & 1 & 1 \\
-1 & 0 & 0 & 1
\end{array}\right], \\
& U_{1-}=U_{4-}=U_{7-}=\left[\begin{array}{llc}
1 & 1 & 1
\end{array}\right], \\
& X_{2}=X_{5}=X_{8}=\left[\begin{array}{cccc}
1 & 0 & 3 & -1 \\
-1 & 2 & -2 & 5
\end{array}\right], \\
& U_{2-}=U_{5-}=U_{8-}=\left[\begin{array}{lll}
1 & 1 & 1
\end{array}\right], \\
& X_{3}=X_{6}=X_{9}=\left[\begin{array}{cccc}
1 & 2 & 0 & -1 \\
-1 & 1 & 2 & 0
\end{array}\right], \\
& U_{3-}=U_{6-}=U_{9-}=\left[\begin{array}{lll}
1 & 1 & 1
\end{array}\right] .
\end{aligned}
$$

It is easy to verify that the data are informative for stabilization by state feedback, and, using directly these data, we compute feedback gains

$$
\begin{aligned}
& K_{1}=K_{4}=K_{7}=\left[\begin{array}{ll}
-0.3677 & -1.3560
\end{array}\right], \\
& K_{2}=K_{5}=K_{8}=\left[\begin{array}{ll}
0.4183 & -1.4385
\end{array}\right], \\
& K_{3}=K_{6}=K_{9}=\left[\begin{array}{ll}
0.0017 & 1.0008
\end{array}\right] .
\end{aligned}
$$

Similarly, we compute solutions $M_{i}$ to the linear equations (19) and (20), and obtain

$$
\begin{aligned}
& M_{1}=M_{4}=M_{7}=\left[\begin{array}{cc}
0 & 1 \\
2 & -1 \\
-1 & 0
\end{array}\right], M_{2}=M_{5}=M_{8}=\left[\begin{array}{cc}
0.4 & -1.4 \\
0.4 & 0.6 \\
0.2 & 0.8
\end{array}\right], \\
& M_{3}=M_{6}=M_{9}=\left[\begin{array}{cc}
0.6 & -0.1 \\
-0.3 & 0.3 \\
0.7 & -0.2
\end{array}\right] .
\end{aligned}
$$




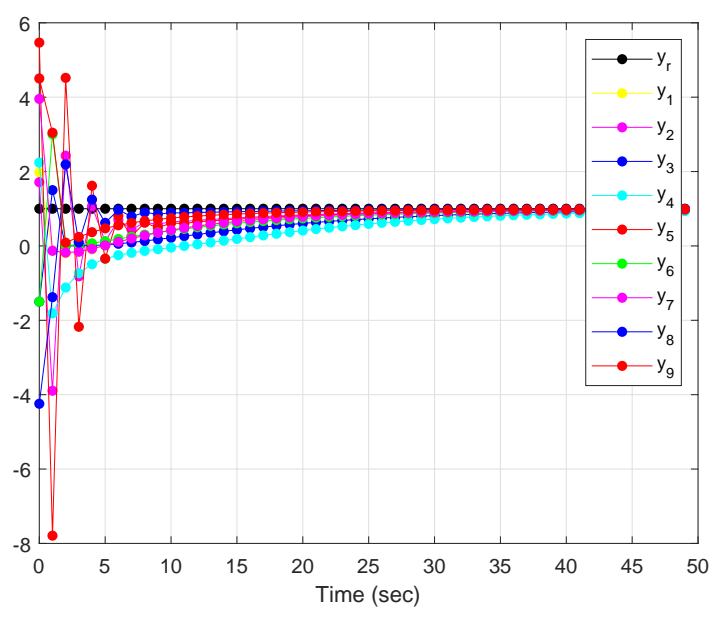

Fig. 2. Plots of the output trajectories $y_{r}, y_{1}, y_{2}, \ldots, y_{9}$.

According to Proposition 6 , since the data $\left(U_{i-}, X_{i}\right)$ are informative for stabilization by state feedback and informative for output regulation, the data are also informative for output synchronization. Subsequently, we compute gain matrices $F$, $\Pi_{i}$ and $\Gamma_{i}$. It is shown in Figure 2 that the associated protocol indeed achieves output synchronization.

\section{CONCLUSIONS AND FUTURE WORK}

In this paper, we have considered an informativity approach to data-driven output synchronization for leaderfollower multi-agent systems. We have provided necessary and sufficient data-based conditions for output synchronization. We have provided a design method for computing such distributed output synchronizing protocols directly from data. We have also extended the results to the special case that the followers are disturbance-free.

As a possibility for future research, we mention the more practical and challenging situation that the external disturbance is unknown [14]. It would also be interesting to extend the results in this paper to the case that only input and output data of the followers are available [13].

\section{REFERENCES}

[1] R. Olfati-Saber and R. M. Murray, "Consensus problems in networks of agents with switching topology and time-delays," IEEE Transactions on Automatic Control, vol. 49, no. 9, pp. 1520-1533, 2004.

[2] P. Wieland, R. Sepulchre, and F. Allgöwer, "An internal model principle is necessary and sufficient for linear output synchronization," Automatica, vol. 47, no. 5, pp. 1068-1074, 2011.

[3] J. Jiao, H. L. Trentelman, and M. K. Camlibel, "A suboptimality approach to distributed $H_{2}$ control by dynamic output feedback," Automatica, vol. 121, p. 109164, 2020.

[4] Z. Li and Z. Duan, Cooperative Control of Multi-Agent Systems: A Consensus Region Approach. CRC Press, 2014.

[5] J. Jiao, H. L. Trentelman, and M. K. Camlibel, "Distributed linear quadratic optimal control: compute locally and act globally," IEEE Control Systems Letters, vol. 4, no. 1, pp. 67-72, 2020.

[6] — "A suboptimality approach to distributed linear quadratic optimal control," IEEE Transactions on Automatic Control, vol. 65, no. 3, pp. 1218-1225, 2020.

[7] X. Li, F. Liu, M. Buss, and S. Hirche, "Fully distributed consensus control for linear multiagent systems: a reduced-order adaptive feedback approach," IEEE Transactions on Control of Network Systems, vol. 7, no. 2, pp. 967-976, 2020.
[8] H. Zhang, H. Jiang, Y. Luo, and G. Xiao, "Data-driven optimal consensus control for discrete-time multi-agent systems with unknown dynamics using reinforcement learning method," IEEE Transactions on Industrial Electronics, vol. 64, no. 5, pp. 4091-4100, 2017.

[9] M. I. Abouheaf, F. L. Lewis, K. G. Vamvoudakis, S. Haesaert, and R. Babuska, "Multi-agent discrete-time graphical games and reinforcement learning solutions," Automatica, vol. 50, no. 12, pp. 3038-3053, 2014

[10] J. C. Willems, P. Rapisarda, I. Markovsky, and B. L. De Moor, "A note on persistency of excitation," Systems \& Control Letters, vol. 54, no. 4, pp. 325-329, 2005.

[11] C. De Persis and P. Tesi, "Formulas for data-driven control: stabilization, optimality, and robustness," IEEE Transactions on Automatic Control, vol. 65, no. 3, pp. 909-924, 2020.

[12] A. Romer, J. Berberich, J. Köhler, and F. Allgöwer, "One-shot verification of dissipativity properties from input-output data," IEEE Control Systems Letters, vol. 3, no. 3, pp. 709-714, 2019.

[13] H. J. van Waarde, J. Eising, H. L. Trentelman, and M. K. Camlibel, "Data informativity: a new perspective on data-driven analysis and control," IEEE Transactions on Automatic Control, vol. 65, no. 11, pp. 4753-4768, 2020.

[14] H. J. van Waarde, M. K. Camlibel, and M. Mesbahi, "From noisy data to feedback controllers: non-conservative design via a matrix SLemma," IEEE Transactions on Automatic Control, pp. 1-1, 2020.

[15] J. Berberich, A. Koch, C. W. Scherer, and F. Allgöwer, "Robust datadriven state-feedback design," in 2020 American Control Conference (ACC), 2020, pp. 1532-1538.

[16] N. Monshizadeh, "Amidst data-driven model reduction and control," IEEE Control Systems Letters, vol. 4, no. 4, pp. 833-838, 2020.

[17] H. J. van Waarde, C. De Persis, M. K. Camlibel, and P. Tesi, "Willems' fundamental lemma for state-space systems and its extension to multiple datasets," IEEE Control Systems Letters, vol. 4, no. 3, pp 602-607, 2020.

[18] A. Allibhoy and J. Cortés, "Data-based receding horizon control of linear network systems," IEEE Control Systems Letters, vol. 5, no. 4, pp. 1207-1212, 2021.

[19] T. R. Steentjes, M. Lazar, and P. M. Van den Hof, "Data-driven distributed control: Virtual reference feedback tuning in dynamic networks," in 2020 59th IEEE Conference on Decision and Control (CDC), 2020, pp. 1804-1809.

[20] T. R. V. Steentjes, M. Lazar, and P. M. J. V. den Hof, "Guaranteed $H_{\infty}$ performance analysis and controller synthesis for interconnected linear systems from noisy input-state data," 2021, [Online]. Available: https://arxiv.org/abs/2103.14399.

[21] G. Baggio, D. S. Bassett, and F. Pasqualetti, "Data-driven control of complex networks," Nature Communications, vol. 12, no. 1, p. 1429 , Mar 2021.

[22] A. Cherukuri and J. Cortés, "Cooperative data-driven distributionally robust optimization," IEEE Transactions on Automatic Control, vol. 65, no. 10, pp. 4400-4407, 2020.

[23] B. Kiumarsi and F. L. Lewis, "Output synchronization of heterogeneous discrete-time systems: a model-free optimal approach," Automatica, vol. 84, pp. 86-94, 2017.

[24] J. Jiao, H. L. Trentelman, and M. K. Camlibel, " $H_{2}$ suboptimal output synchronization of heterogeneous multi-agent systems," Systems \& Control Letters, vol. 149, p. 104872, 2021.

[25] T. Yang, X. Wang, A. Saberi, and A. A. Stoorvogel, "Output synchronization for heterogeneous networks of discrete-time introspective right-invertible agents with uniform constant communication delay," in 2013 American Control Conference, 2013, pp. 516-521.

[26] Y. Jiang, J. Fan, W. Gao, T. Chai, and F. L. Lewis, "Cooperative adaptive optimal output regulation of nonlinear discrete-time multiagent systems," Automatica, vol. 121, p. 109149, 2020.

[27] K. Hengster-Movric, K. You, F. L. Lewis, and L. Xie, "Synchronization of discrete-time multi-agent systems on graphs using riccati design," Automatica, vol. 49, no. 2, pp. 414 - 423, 2013.

[28] H. J. van Waarde and M. Mesbahi, "Data-driven parameterizations of suboptimal LQR and $\mathrm{H}_{2}$ controllers," in IFAC 2020, 2020.

[29] H. L. Trentelman, H. J. van Waarde, and M. K. Camlibel, "An informativity approach to data-driven tracking and regulation," 2020, [Online]. Available: https://arxiv.org/abs/0801.3390. 